\title{
SOP Autonome Störungen
}

Das autonome Nervensystem steuert unwillkürlich zahlreiche Vorgänge im menschlichen Körper. Neben der Kreislaufregulation werden unter anderem auch Magen-Darm-Tätigkeit, Blasenentleerung und die Schweißsekretion durch das Zusammenwirken von Parasympathikus und Sympathikus gesteuert. Anatomische Strukturen sind der sympathische Grenzstrang (Tractus sympathicus), die intramuralen Nervengeflechte im Brustraum, in den Bauchorganen und den Beckenorganen sowie den Ganglien des N. oculomotorius, des N. facialis, des N. glossopharyngeus und des N. vagus. Erkrankungen des autonomen Nervensystems können sowohl isoliert als autonome Störungen) auftreten als auch im Rahmen von neurologischen oder internistischen Systemerkrankungen.

\section{Allgemeines Vorgehen - von der Diagnose zur Therapie}

Die vegetative Anamnese umfasst mindestens Fragen zu orthostatischen Beschwerden (Herzrasen, Schwindel beim Aufrichten, Blässe), Schweißsekretion, zur Blasenentleerung/-kontinenz, Stuhlfrequenz/-beschaffenheit (Obstipation vs. Diarrhöen), Libido/Erektion und Schlaf. Zudem sollten detailliert tageszeitliche Abhängigkeit, Dauer der Symptome, Ernährungs- und Trinkgewohnheiten, sportliche Betätigung erfragt wer- den. Das Flussdiagramm $>$ Abb. 1 fasst den Weg zur Diagnose autonomer Störungen zusammen.

\section{Spezielle Krankheitsbilder}

\section{Passagerer Bewusstseinsverlust - Transient Loss of Consciousness (TLOC)}

Der passagere Bewusstseinsverlust ist durch eine vorübergehende, meist rasch reversible, quantitative Bewusstseinsstörung definiert. Diese geht üblicherweise nicht mit einer bleibenden Schädigung einher. Ursäch-

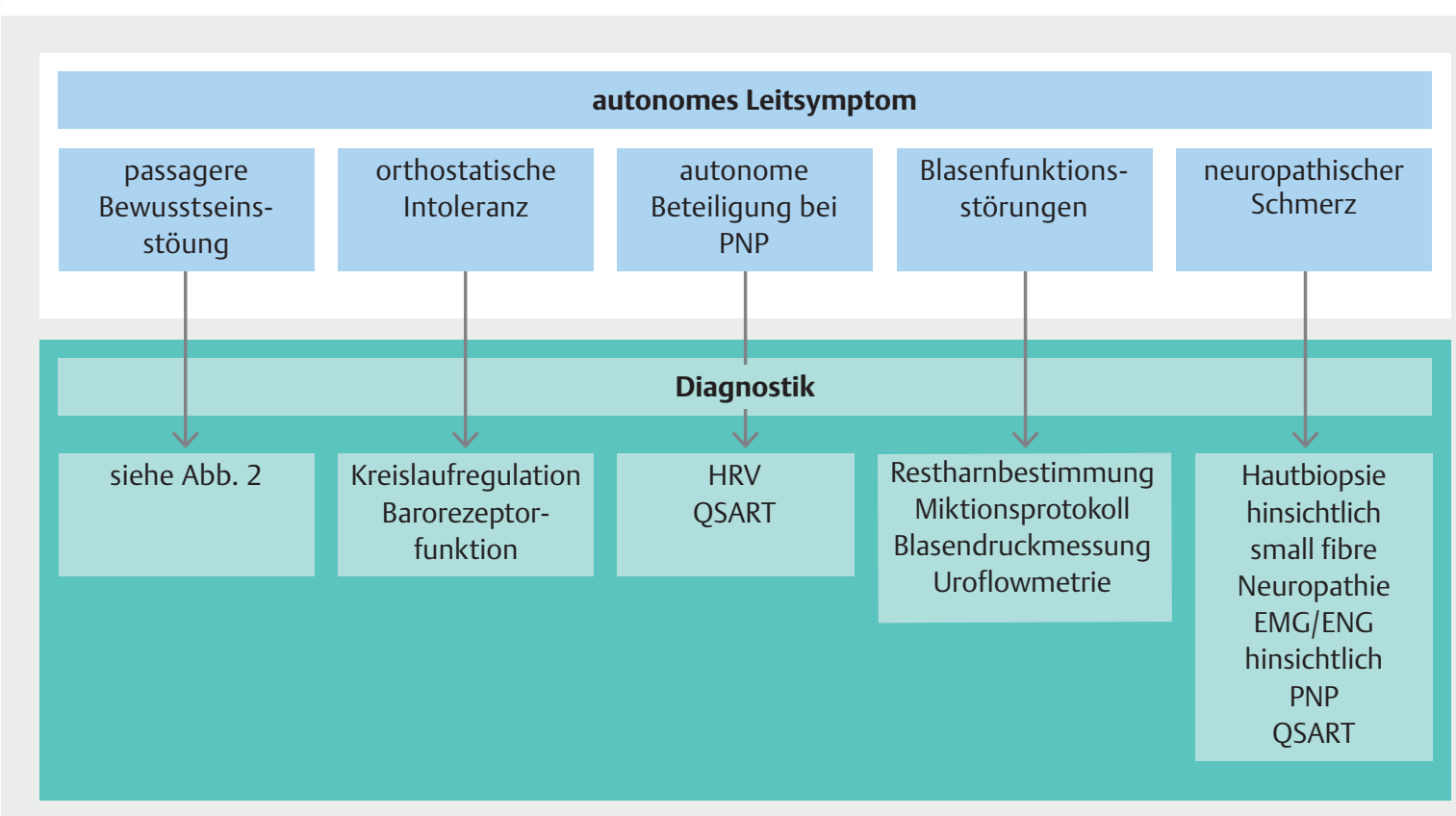

Abb. 1 Autonome Störungen - allgemeines Vorgehen. 
lich sind neben kreislaufabhängigen Störungen, siehe Synkopen, auch verschiedene andere Erkrankungen aus dem neurologischen und internistischen Fachgebiet. Differenzialdiagnostisch abzugrenzen sind epileptische Anfälle, psychogene nicht-epileptische Anfälle, metabolische Entgleisungen (Elektrolyte, Blutzucker, Ammoniak), Intoxikationen oder eine TIA im vertebrobasilären Stromgebiet.

\section{Synkopen}

Unter einer Synkope versteht man einen plötzlich eintretenden, passageren Verlust von Bewusstsein und Muskeltonus, bedingt durch eine zerebrale Minderdurchblutung. Meist fällt dabei der systolische Blutdruck unter $70 \mathrm{mmHg}$. Ursächlich können sowohl primär ein Abfall des Blutdruckes als auch ein Abfall der Herzfrequenz bzw. eine Pause sein. Klinisch ist die Synkope durch eine rasche Erholung - im Gegensatz zum epileptischen Anfall - charakterisiert. Einen Überblick und einen diagnostischen Algorithmus bietet - Abb. 2 . Prodromi kommen häufig, jedoch nicht immer vor und umfassen typischerweise einen Schweißausbruch, "Schwarzwerden vor den Augen“, Übelkeit und eine möglicherweise fremdanamnestisch beobachtete Blässe. Myoklonien treten in mehr als 50 \% der Fälle auf.

\section{Orthostatische Intoleranz}

Für das Vorliegen einer orthostatischen Intoleranz können sowohl Veränderungen der Blutdruckregulation als auch der Herzfrequenz ursächlich sein. Patienten schildern, dass die Symptome typischerweise im Stehen, selten auch im Sitzen auftreten. Im Liegen treten typischerweise keine Beschwerden auf.

\section{Orthostatische Hypotonie}

Die orthostatische Hyptonie ist definiert durch einen anhaltenden systolischen Blutdruckabfall nach 3 Min. im Stand im Gegensatz zum Ruhewert im Liegen. Dabei sind als Grenzwerte ein Abfall des systolischen Blutdrucks um $20 \mathrm{mmHg}$ und/oder ein Abfall des diastolischen Blutdrucks um $10 \mathrm{mmHg}$ zwischen Ruhe und Stand definiert worden. Einen Überblick über die Interpretation von Befunden in der Langzeit-Blutdruckmessung findet sich im Flussdiagramm - Abb. 3.

\section{Posturales orthostatisches Tachykardiesyndrom}

Das posturale orthostatische Tachykardiesyndrom (POTS) ist durch einen pathologisch hohen Anstieg der Herzfrequenz im Stehen gegenüber dem Ruhewert und durch das Vorliegen von Symptomen einer orthostatischen Intoleranz definiert. Dabei wird für Erwachsene ein Grenzwert von mehr als 30 Schlägen pro Min. und für Kinder und Jugendliche ein Grenzwert von mehr als 40 Schlägen pro Minute festgelegt. Alternativ kann ein POTS bei einer Herzfrequenz im Stehen von über 120/ min diagnostiziert werden. Gefordert werden eine aus- reichende Hydratation und der fehlende Konsum von Stimulanzien (u.a. Koffein, Nikotin und ähnlichem). Kommt es im Rahmen des POTS zu Synkopen, sind diese typischerweise neurokardiogene Synkopen.

Neurokardiogene Synkopen

Neurokardiogene Synkopen sind durch einen progredienten Blutdruckabfall mit begleitendem Herzfrequenzabfall gekennzeichnet. Hierbei kommt es zu einer raschen Zunahme des Parasympathikotonus mit einer konsekutiven Bradykardie und zu einer Abnahme des Sympathikotonus mit einer daraus resultierenden peripheren Vasodilatation, die zu einem systolischen Blutdruckabfall führt. Ursachen für neurokardiogene Synkopen können sowohl zentralnervös verarbeitete Reize sein, wie Schmerz, Ekel oder Angst, als auch eine Dysfunktion des Baroreflexes, ausgelöst durch Miktion, Defäkation, Husten, Schlucken u. v. m.

\section{Parkinson-Syndrome}

Sowohl das idiopathische Parkinsonsyndrom als auch die Multisystematrophie (MSA) sind häufig von autonomen Störungen begleitet. Neben der häufig vorkommenden orthostatischen Hypotonie sind auch Blasenentleerungsstörungen und Störungen der Motilität des Gastrointestinaltraktes (verzögerte Magenentleerung, Obstipation) sowie sexuelle Funktionsstörungen regelhaft und schon in frühen Stadien der Erkrankung zu finden.

\section{Diabetes mellitus}

Neben der typischen sensomotorischen Neuropathie kommt es beim Diabetes mellitus, abhängig von Krankheitsdauer und Ausprägung der Hyperglykämie, zu autonomen Störungen. Dabei können insbesondere die Autoregulation des Schwitzens als auch die Funktion unmyelinisierter C-Fasern mit einer schmerzhaften Neuropathie betroffen sein. Eine Ruhetachykardie, verminderte Herzfrequenzvariabilität oder verminderte Herzwahrnehmung sind typische Zeichen einer kardialen diabetischen autonomen Neuropathie (KADN).

\section{Guillain-Barré-Syndrom}

Das Guillain-Barré-Syndrom (GBS) ist eine akut bis subakut verlaufende Polyradikuloneuritis, hierbei können neben den typischerweise initial auftretenden Sensibilitätsstörungen, den aufsteigenden Paresen und den Reflexausfällen im Krankheitsverlauf exzessive autonome Störungen auftreten. Diese können sowohl bradykarde als auch tachykarde Herzrhythmusstörungen, Blutdruckabfälle und exzessive Entgleisungen wie auch Störungen des Schwitzens sowie Funktionsstörungen von Blase und Darm sein. Insbesondere die kardialen Veränderungen können potenziell zu lebensbedrohlichen Komplikationen führen. 


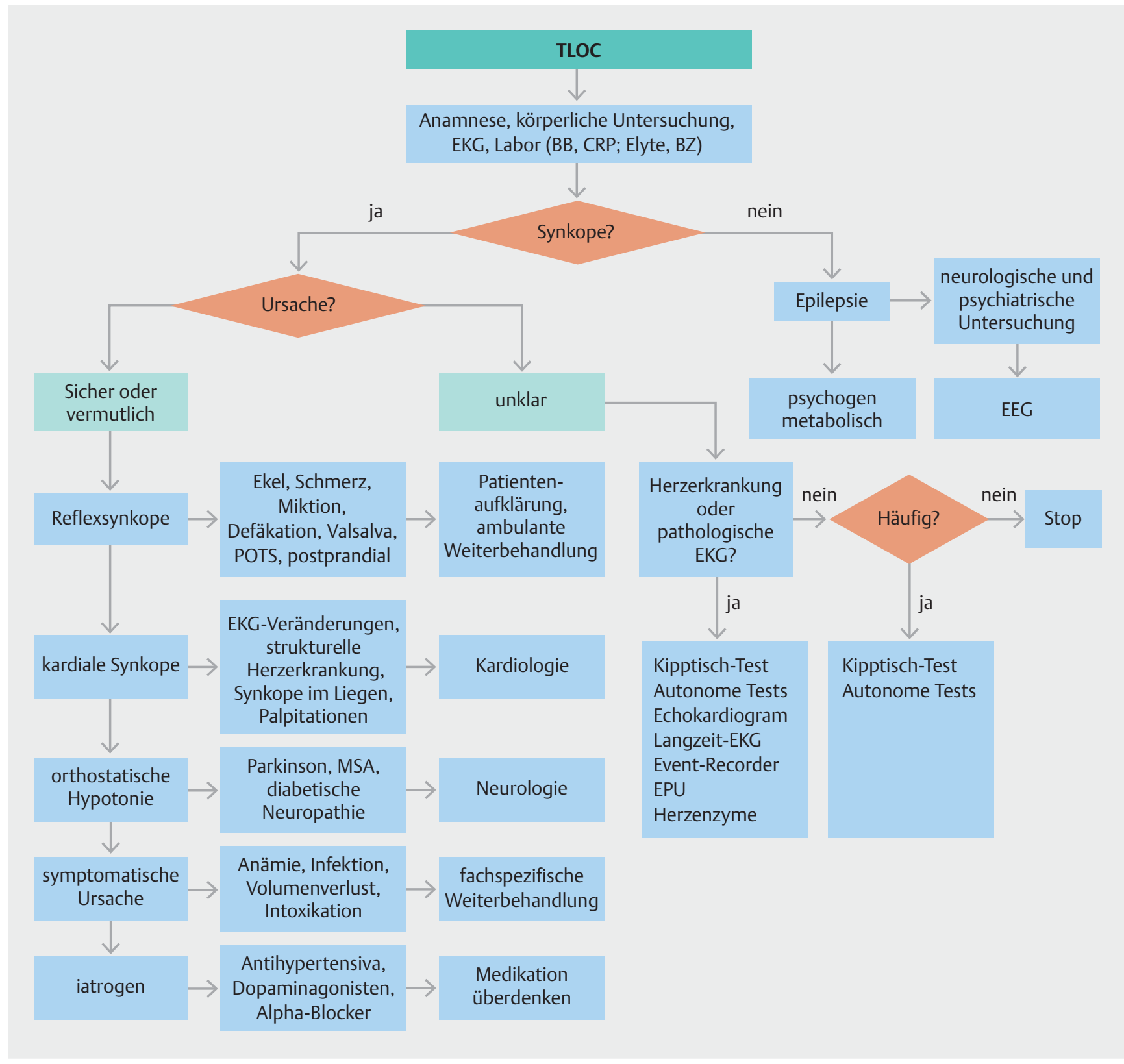

Abb. 2 Synkopen: Überblick und Diagnose.

\section{Neurogene Blasenentleerungsstörungen}

Zahlreiche neurologische Erkrankungen können zu Blasenentleerungsstörungen führen. Diese treten typischerweise bei der Multiplen Sklerose, bei neurodegenerativen und bei zerebrovaskulären Erkrankungen auf und umfassen die Detrusorhyperaktivität, die Detrusor-Sphinkter-Dyssynergie sowie die Detrusor- und Sphinkterhypoaktivität. Blasenentleerungsstörungen treten auch im Rahmen von Polyneuropathien auf und gehen dann mit einer erhöhten Restharnbildung einher.

\section{Small-Fiber-Neuropathie}

Hierbei sind die feinsten und kleinsten Nervenfasern in der Regel ohne eine wesentliche Beteiligung der somatosensiblen Fasern betroffen. Klinisch stehen typischerweise ein brennender Schmerz und eine Schweißsekretionsstörung im Vordergrund. Die histologische Untersuchung somatosensibler Fasern zeigt selten pathologische Befunde, diagnostisch ist eine Hautbiopsie zum Nachweis eines Verlusts kleiner Nervenfasern wegweisend. 


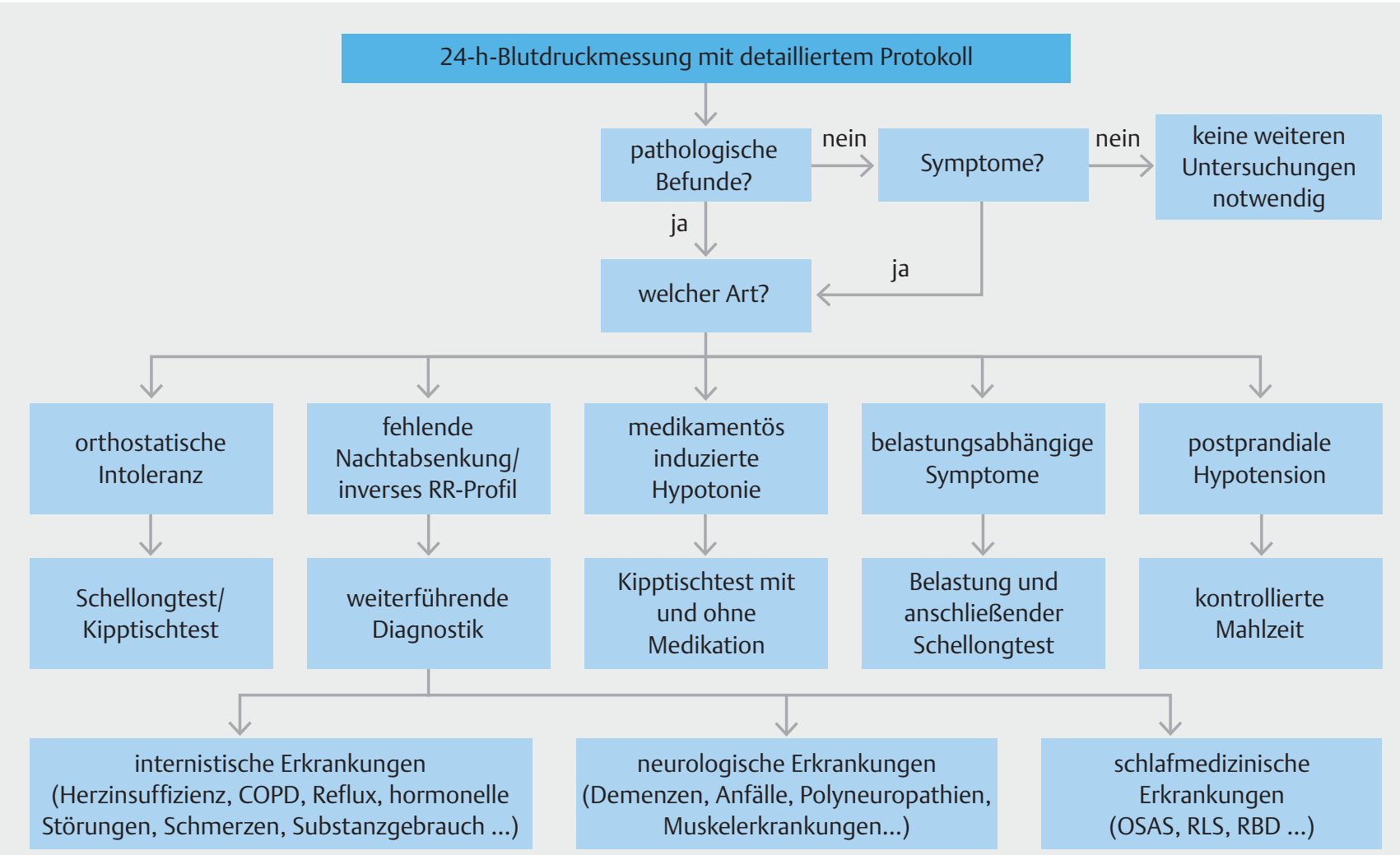

- Abb. 3 Interpretation von Befunden in der Langzeit-Blutdruckmessung. Interpretation von 24-Stunden-Blutdruckmessungen, adaptiert nach Stübner 2013. RR: arterieller Blutdruck, COPD: Chronisch obstruktive Lungenerkrankung, OSAS: Obstruktives Schlafapnoesyndrom, RLS: RestlessLegsSyndrom, RBD: REM-Schlaf-Verhaltensstörung Quelle: nach Stuebner et al. Twenty-Four Hour non-invasive ambulatory blood pressure and heart rate monitoring in Parkinson's disease. Front Neurol 2013;4:49.

\section{Interessenkonflikt}

Die Autorinnen/Autoren geben an, dass kein Interessenkonflikt besteht.

\section{Autorinnen/Autoren}

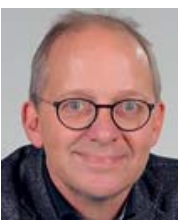

\section{Prof. Dr. med. Carl-Albrecht Haensch}

Nach Medizinstudium in Düsseldorf und Dissertation am Diabetes Forschungs-Institut zur Immunpathogenese der diabetischen Neuropathie, Facharztausbildung an der Klinik für Neurologie und klinische Neurophysiologie Wuppertal. Facharzt für Neurologie, Schlafmedizin und Spezielle Schmerztherapie. Habilitation an der Universität Witten/Herdecke zur Diagnostik des autonomen Nervensystems. Beirat der Arbeitsgemeinschaft „Autonomes Nervensystem“ der DGN. Seit 2006 apl. Professur für Neurologie der Universität Witten/Herdecke. 2010 Robert Wartenberg-Preis der DGN. Seit 2014 Chefarzt der Klinik für Neurologie der Kliniken Maria Hilf Mönchengladbach. Autor und Koordinator verschiedener Leitlinien zu Themen des Autonomen Nervensystems. Herausgeber der Fachzeitschrift „Klinischen Neurophysiologie“.

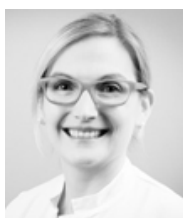

\section{Dr. med. Anke Lührs}

Medizinstudium an der Medizinischen Hochschule Hannover, Dissertation in der dortigen Klinik für Neurologie zur Quantifizierung von Feinmotorikstörungen bei Parkinson-Patienten. Facharztausbildung in der Klinik für Neurologie und klinische Neurophysiologie in Wuppertal bei Prof. Dr. S. Isenmann. Oberärztin an der Klinik für Neurologie der Kliniken Maria Hilf Mönchengladbach, Aufbau und Leitung des dortigen Autonomen Labors. Zusatzweiterbildung Geriatrie - Oberärztin am Klinikum Braunschweig mit Schwerpunkt Neurogeriatrie. Seit April 2019 Leitende Oberärztin der Klinik für Neurologie und klinische Neurophysiologie am St. Josef Krankenhaus in Moers.

\section{Korrespondenzadresse}

Prof. Dr. med. Carl-Albrecht Haensch

Klinik für Neurologie

Kliniken Maria Hilf GmbH

Akademisches Lehrkrankenhaus der Uniklinik RWTH Aachen Viersener Straße 450

41063 Mönchengladbach

E-Mail: Carl-Albrecht.Haensch@mariahilf.de 
Zitierweise für diesen Artikel

Haensch C-A, Lührs A. Autonome Störungen. In: Bischoff C, Buchner H, Hrsg. SOPs Neurophysiologische Diagnostik, 1. Auflage. Stuttgart: Thieme 2018: 124-127.

\section{Literatur}

[1] Haensch CA. Synkopen. In: Buchner H, Noth ], Heinze H] , Hrsg. Klinische Elektroneurophysiologie: Evozierte Potentiale - Vegetative Funktionsdiagnostik - Elektrookulographie. Referenzreihe Neurologie. Stuttgart: Thieme; 2005: 207-214

[2] Haensch CA, Jost W, Hrsg. Das Autonome Nervensystem. Stuttgart: Kohlhammer; 2009
[3] Haensch CA. Orthostatische Dysregulation und primär autonome Störungen. In: Bischoff C, Straube A, Hrsg. Leitlinien Klinische Neurophysiologie. Stuttgart: Kohlhammer; 2014: 281-288

[4] Stuebner E, Vichayanrat E, Low DA et al. Twenty-four hour non-invasive ambulatory blood pressure and heart rate monitoring in Parkinson's disease. Front Neurol 2013; 4: 49

\section{Bibliografie}

DOI https://doi.org/10.1055/a-1022-2399

Neurologie up2date 2020; 3: 6-11

(c) Georg Thieme Verlag KG Stuttgart · New York

ISSN 2511-3453 